\title{
Conformational Studies by Dynamic NMR. 98. Stereodynamics of Bond Rotation in $\beta$-Hydroxyesters
}

Giuseppe Bartoli, Lodovico Lunazzi, Massimo Massaccesi and Andrea Mazzanti* Department of Organic Chemistry "A.Mangini”, University of Bologna, Viale Risorgimento 4, Bologna 40136, Italy

mazzand@ms.fci.unibo.it

Page S2 Temperature dependence of the ${ }^{13} \mathrm{C}$ NMR signal of the $\mathrm{CH}_{\alpha}$ carbon of 3 .

Page S3 gHSQC spectrum of 3 at $-43^{\circ} \mathrm{C}$

Page S4: $\quad$ MMX computed energy map of $\mathbf{1}$

Page S5: $\quad$ MMX data of compound 1, conformation -sc

Page S6: $\quad$ MMX data of compound 1, conformation + sc

Page S7: $\quad$ MMX data of compound 1, conformation ap

Page S8: $\quad$ MMX data of compound 3, conformation -sc

Page S9: $\quad$ MMX data of compound 3, conformation + sc

Page S10: MMX data of compound 3, conformation ap

Page S11: $\quad$ MMX data of compound 4, conformation -sc

Page S12: $\quad$ MMX data of compound 4, conformation + sc

Page S13: $\quad$ MMX data of compound 4, conformation ap 
Temperature dependence of the ${ }^{13} \mathrm{C}$ NMR signal of the $\mathrm{CH}_{\alpha}$ carbon of $\mathbf{3}$.

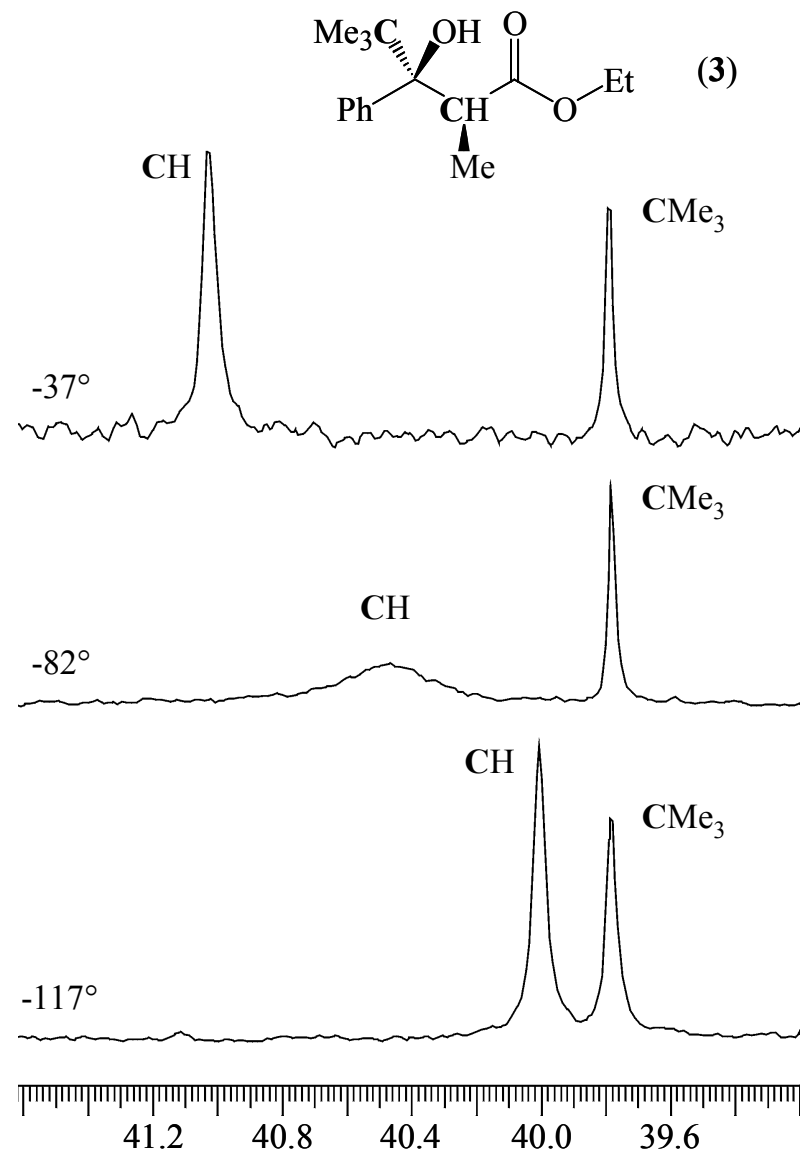




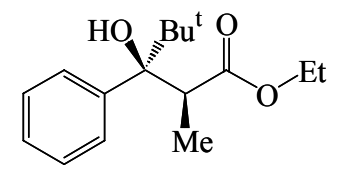

(3)

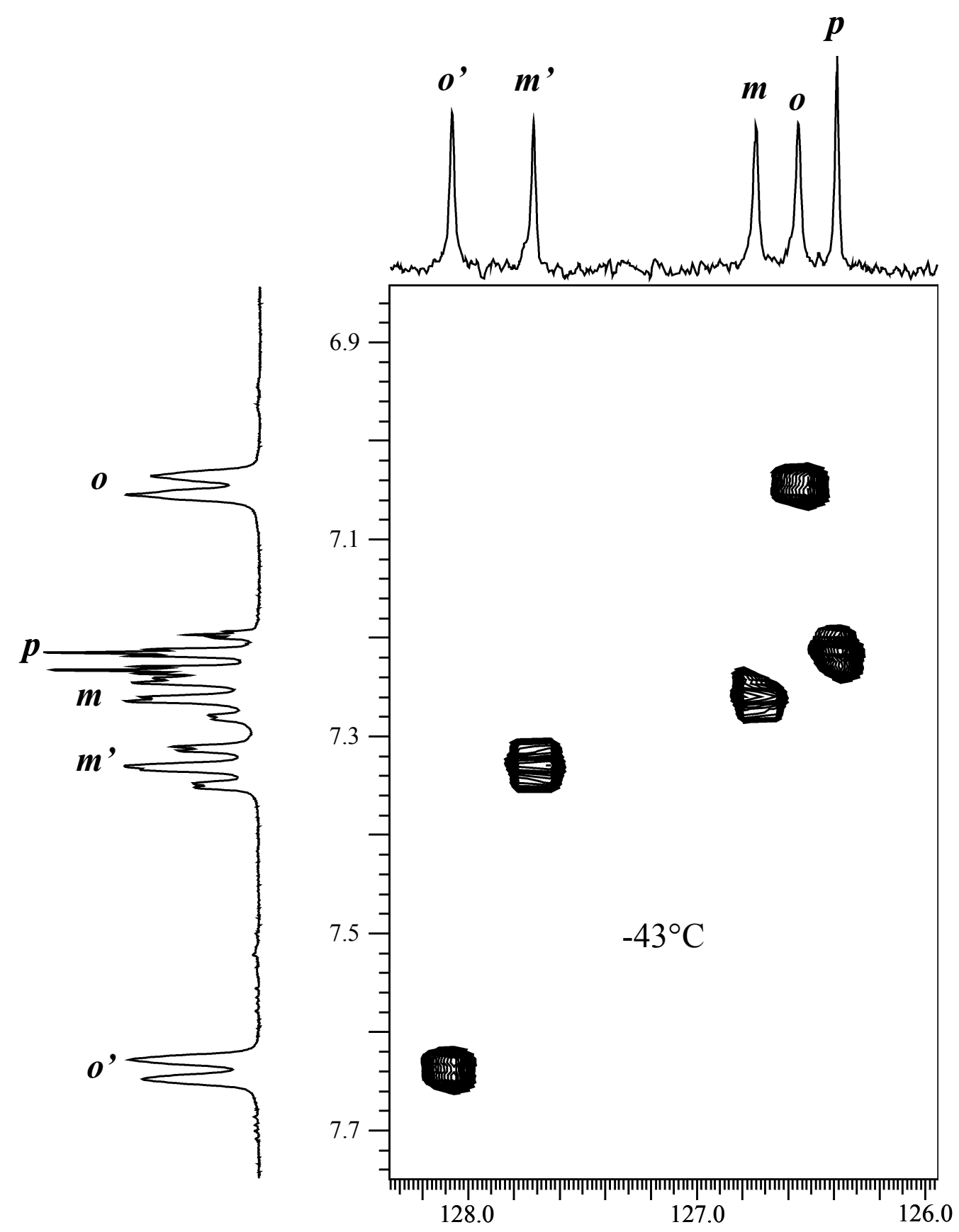

gHSQC Spectrum of 3 at $-43^{\circ} \mathrm{C}$ 

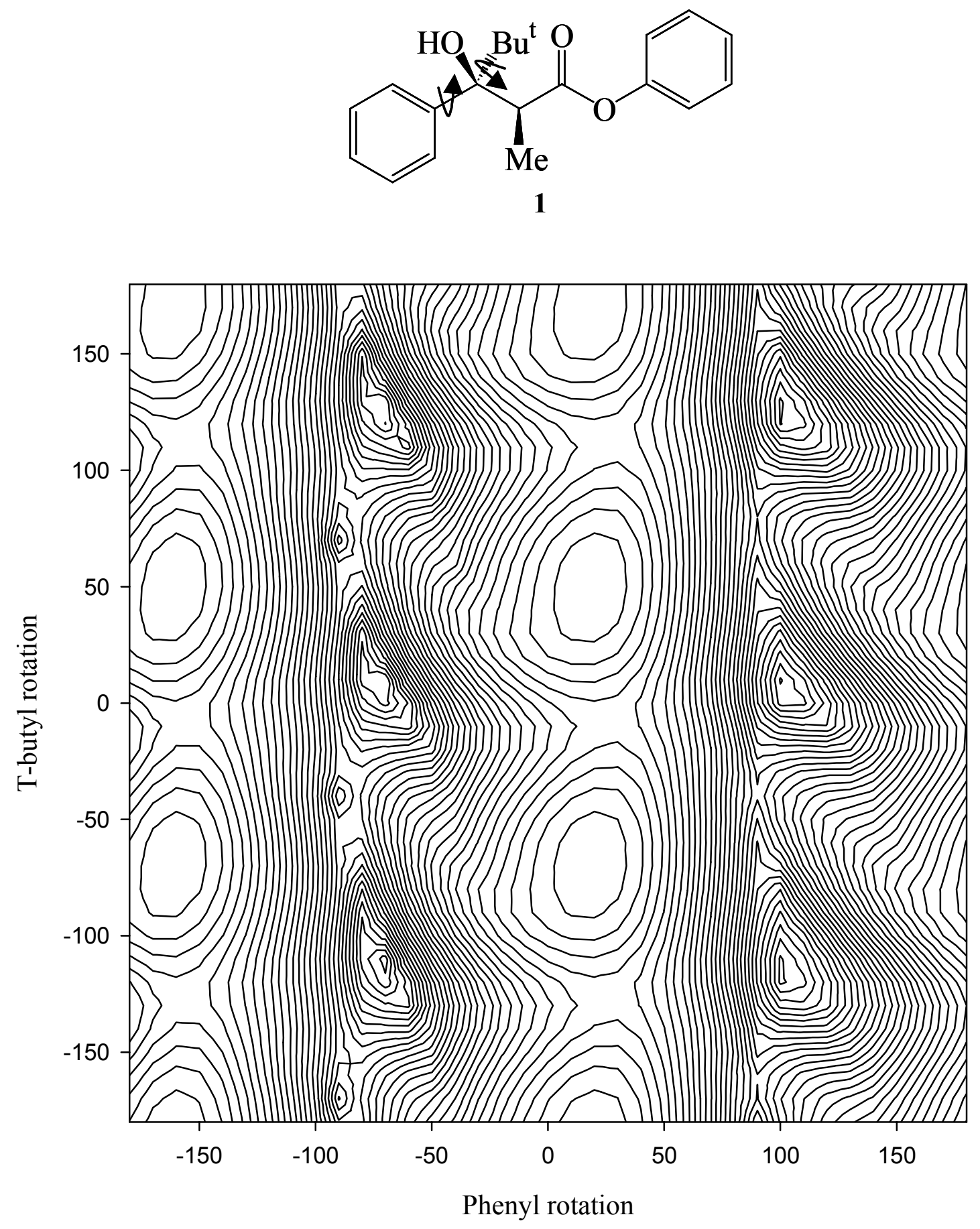

Potential energy map of $\mathbf{1}$, computed as function of the Ar-C and t-Bu-C dihedral angles; the lines are separated by $1 \mathrm{kcal} / \mathrm{mol}$. 
Product 1 -conformation A

Atom

$\mathrm{X}$

$\mathrm{Y}$

Z

\begin{tabular}{|c|c|c|c|c|}
\hline C & 6.0 & -3.346446 & -1.309097 & 2.648818 \\
\hline C & 6.0 & -4.286417 & -2.338223 & 2.776404 \\
\hline $\mathrm{C}$ & 6.0 & -4.584524 & -3.155931 & 1.682783 \\
\hline$C$ & 6.0 & -3.935959 & -2.933235 & 0.465098 \\
\hline$C$ & 6.0 & -2.996767 & -1.901890 & 0.345876 \\
\hline C & 6.0 & -2.679245 & -1.068548 & 1.433473 \\
\hline C & 6.0 & -1.653582 & 0.067392 & 1.311220 \\
\hline C & 6.0 & -0.383705 & -0.449861 & 0.564749 \\
\hline C & 6.0 & 0.771672 & 0.549660 & 0.531416 \\
\hline 0 & 8.0 & 1.309157 & 0.647869 & -0.700908 \\
\hline 0 & 8.0 & 1.149861 & 1.167297 & 1.501890 \\
\hline C & 6.0 & 2.442636 & 2.785550 & -0.719261 \\
\hline $\mathrm{C}$ & 6.0 & 2.313887 & 1.455633 & -1.147230 \\
\hline C & 6.0 & 3.228779 & 0.949628 & -2.082104 \\
\hline C & 6.0 & 4.268485 & 1.750117 & -2.568270 \\
\hline C & 6.0 & 4.398288 & 3.071530 & -2.126667 \\
\hline C & 6.0 & 3.482419 & 3.588085 & -1.203359 \\
\hline O & 8.0 & -1.222750 & 0.415251 & 2.624901 \\
\hline C & 6.0 & 0.188918 & -1.742519 & 1.184997 \\
\hline C & 6.0 & -2.352546 & 1.332843 & 0.682733 \\
\hline $\mathrm{H}$ & 1.0 & -0.417704 & 0.913249 & 2.579526 \\
\hline $\mathrm{C}$ & 6.0 & -3.683409 & 1.641611 & 1.417137 \\
\hline C & 6.0 & -2.696656 & 1.160076 & -0.814905 \\
\hline $\mathrm{C}$ & 6.0 & -1.497041 & 2.619034 & 0.786799 \\
\hline $\mathrm{H}$ & 1.0 & -3.150470 & -0.687274 & 3.536941 \\
\hline $\mathrm{H}$ & 1.0 & -4.794970 & -2.504972 & 3.741192 \\
\hline $\mathrm{H}$ & 1.0 & -5.324822 & -3.967913 & 1.779827 \\
\hline $\mathrm{H}$ & 1.0 & -4.166360 & -3.573829 & -0.403187 \\
\hline $\mathrm{H}$ & 1.0 & -2.516154 & -1.772883 & -0.635494 \\
\hline $\mathrm{H}$ & 1.0 & -0.652677 & -0.686630 & -0.489042 \\
\hline $\mathrm{H}$ & 1.0 & 1.728133 & 3.222599 & -0.003352 \\
\hline $\mathrm{H}$ & 1.0 & 3.131679 & -0.090809 & -2.435381 \\
\hline $\mathrm{H}$ & 1.0 & 4.985577 & 1.340085 & -3.299424 \\
\hline $\mathrm{H}$ & 1.0 & 5.217569 & 3.704586 & -2.507314 \\
\hline $\mathrm{H}$ & 1.0 & 3.579173 & 4.631531 & -0.858511 \\
\hline $\mathrm{H}$ & 1.0 & 0.507549 & -1.589394 & 2.241225 \\
\hline $\mathrm{H}$ & 1.0 & -0.537874 & -2.585193 & 1.173648 \\
\hline $\mathrm{H}$ & 1.0 & 1.083524 & -2.091746 & 0.618759 \\
\hline $\mathrm{H}$ & 1.0 & -4.433716 & 0.828718 & 1.294593 \\
\hline $\mathrm{H}$ & 1.0 & -3.528659 & 1.806240 & 2.507365 \\
\hline $\mathrm{H}$ & 1.0 & -4.169447 & 2.562239 & 1.020711 \\
\hline $\mathrm{H}$ & 1.0 & -3.422573 & 0.336973 & -0.991151 \\
\hline $\mathrm{H}$ & 1.0 & -3.173510 & 2.078076 & -1.228174 \\
\hline $\mathrm{H}$ & 1.0 & -1.793704 & 0.972486 & -1.437291 \\
\hline $\mathrm{H}$ & 1.0 & -1.163870 & 2.830920 & 1.827222 \\
\hline $\mathrm{H}$ & 1.0 & -0.603598 & 2.579891 & 0.128600 \\
\hline $\mathrm{H}$ & 1.0 & -2.064126 & 3.517576 & 0.452624 \\
\hline
\end{tabular}


Product 1 -conformation $B$

Atom

$\mathrm{X}$

Y

Z

\begin{tabular}{|c|c|c|c|c|}
\hline C & 6.0 & -2.356669 & 0.450692 & -1.980663 \\
\hline C & 6.0 & -3.459435 & -0.398283 & -1.829101 \\
\hline C & 6.0 & -3.273727 & -1.778025 & -1.707163 \\
\hline C & 6.0 & -1.977544 & -2.297130 & -1.752769 \\
\hline C & 6.0 & -0.882615 & -1.438975 & -1.909780 \\
\hline C & 6.0 & -1.039376 & -0.046029 & -2.003705 \\
\hline C & 6.0 & 0.168869 & 0.893555 & -2.119542 \\
\hline C & 6.0 & 1.223941 & 0.574459 & -1.002588 \\
\hline C & 6.0 & 0.465218 & 0.388678 & 0.316199 \\
\hline 0 & 8.0 & 0.665507 & -0.821172 & 0.871891 \\
\hline O & 8.0 & -0.261689 & 1.239112 & 0.781995 \\
\hline C & 6.0 & 0.198861 & -0.516473 & 3.219082 \\
\hline C & 6.0 & 0.178528 & -1.296310 & 2.053467 \\
\hline C & 6.0 & -0.317768 & -2.606189 & 2.112797 \\
\hline C & 6.0 & -0.821500 & -3.120489 & 3.312885 \\
\hline C & 6.0 & -0.822154 & -2.328939 & 4.467035 \\
\hline C & 6.0 & -0.308929 & -1.027629 & 4.419164 \\
\hline O & 8.0 & -0.252240 & 2.223853 & -1.797354 \\
\hline C & 6.0 & 2.360472 & -0.443324 & -1.219176 \\
\hline C & 6.0 & 0.665280 & 0.981226 & -3.608456 \\
\hline $\mathrm{H}$ & 1.0 & -0.621386 & 2.223520 & -0.923409 \\
\hline C & 6.0 & -0.454545 & 1.552559 & -4.522723 \\
\hline C & 6.0 & 1.037951 & -0.379300 & -4.240889 \\
\hline C & 6.0 & 1.867365 & 1.945687 & -3.748271 \\
\hline $\mathrm{H}$ & 1.0 & -2.570297 & 1.526334 & -2.081920 \\
\hline $\mathrm{H}$ & 1.0 & -4.479968 & 0.020652 & -1.805675 \\
\hline $\mathrm{H}$ & 1.0 & -4.140101 & -2.449293 & -1.581146 \\
\hline $\mathrm{H}$ & 1.0 & -1.819234 & -3.385604 & -1.666606 \\
\hline $\mathrm{H}$ & 1.0 & 0.109493 & -1.903908 & -1.960000 \\
\hline $\mathrm{H}$ & 1.0 & 1.800078 & 1.515729 & -0.826493 \\
\hline $\mathrm{H}$ & 1.0 & 0.620012 & 0.502409 & 3.204532 \\
\hline $\mathrm{H}$ & 1.0 & -0.319354 & -3.232626 & 1.204744 \\
\hline $\mathrm{H}$ & 1.0 & -1.218846 & -4.148922 & 3.348702 \\
\hline $\mathrm{H}$ & 1.0 & -1.222917 & -2.730855 & 5.412901 \\
\hline $\mathrm{H}$ & 1.0 & -0.301231 & -0.405288 & 5.329986 \\
\hline $\mathrm{H}$ & 1.0 & 2.017823 & -1.481497 & -1.409721 \\
\hline $\mathrm{H}$ & 1.0 & 3.051303 & -0.160040 & -2.041893 \\
\hline $\mathrm{H}$ & 1.0 & 3.013841 & -0.495536 & -0.316938 \\
\hline $\mathrm{H}$ & 1.0 & -1.366964 & 0.914915 & -4.514396 \\
\hline $\mathrm{H}$ & 1.0 & -0.761186 & 2.583902 & -4.237454 \\
\hline $\mathrm{H}$ & 1.0 & -0.123977 & 1.615251 & -5.584821 \\
\hline $\mathrm{H}$ & 1.0 & 0.161909 & -1.060874 & -4.316703 \\
\hline $\mathrm{H}$ & 1.0 & 1.417057 & -0.250905 & -5.280457 \\
\hline $\mathrm{H}$ & 1.0 & 1.838005 & -0.918023 & -3.694743 \\
\hline $\mathrm{H}$ & 1.0 & 1.620553 & 2.974048 & -3.400526 \\
\hline $\mathrm{H}$ & 1.0 & 2.757643 & 1.611917 & -3.175387 \\
\hline $\mathrm{H}$ & 1.0 & 2.194501 & 2.038707 & -4.808989 \\
\hline
\end{tabular}


Product 1 -conformation C Atom $\mathrm{X}$ $\mathrm{Y}$ Z

\begin{tabular}{|c|c|c|c|c|}
\hline C & 6.0 & -1.159050 & 2.994850 & -2.970900 \\
\hline C & 6.0 & -2.475750 & 2.862750 & -3.427700 \\
\hline $\mathrm{C}$ & 6.0 & -3.238250 & 1.749050 & -3.066000 \\
\hline C & 6.0 & -2.663950 & 0.766850 & -2.255700 \\
\hline $\mathrm{C}$ & 6.0 & -1.345050 & 0.908150 & -1.808400 \\
\hline $\mathrm{C}$ & 6.0 & -0.565150 & 2.031250 & -2.131900 \\
\hline C & 6.0 & 0.869150 & 2.199150 & -1.598600 \\
\hline C & 6.0 & 0.849650 & 2.176950 & -0.028000 \\
\hline C & 6.0 & 0.717450 & 0.843750 & 0.704400 \\
\hline 0 & 8.0 & 1.920450 & 0.280850 & 0.922900 \\
\hline 0 & 8.0 & -0.329050 & 0.359150 & 1.074400 \\
\hline $\mathrm{C}$ & 6.0 & 1.619250 & -1.151550 & 2.841600 \\
\hline C & 6.0 & 2.223350 & -0.856550 & 1.610700 \\
\hline $\mathrm{C}$ & 6.0 & 3.192050 & -1.729650 & 1.095500 \\
\hline $\mathrm{C}$ & 6.0 & 3.522450 & -2.905850 & 1.778000 \\
\hline C & 6.0 & 2.893550 & -3.210550 & 2.990700 \\
\hline C & 6.0 & 1.944450 & -2.330250 & 3.523100 \\
\hline O & 8.0 & 1.313950 & 3.516850 & -1.918800 \\
\hline $\mathrm{C}$ & 6.0 & 1.899550 & 3.052050 & 0.697900 \\
\hline $\mathrm{C}$ & 6.0 & 1.851850 & 1.245450 & -2.379900 \\
\hline $\mathrm{H}$ & 1.0 & 0.703550 & 4.134250 & -1.550300 \\
\hline C & 6.0 & 1.819650 & 1.579650 & -3.898600 \\
\hline C & 6.0 & 1.520450 & -0.262450 & -2.294600 \\
\hline C & 6.0 & 3.324050 & 1.426150 & -1.940200 \\
\hline $\mathrm{H}$ & 1.0 & -0.612950 & 3.889750 & -3.307200 \\
\hline $\mathrm{H}$ & 1.0 & -2.915950 & 3.638350 & -4.077600 \\
\hline $\mathrm{H}$ & 1.0 & -4.277650 & 1.643950 & -3.420000 \\
\hline $\mathrm{H}$ & 1.0 & -3.251550 & -0.122850 & -1.971600 \\
\hline $\mathrm{H}$ & 1.0 & -0.941250 & 0.091950 & -1.195400 \\
\hline $\mathrm{H}$ & 1.0 & -0.104250 & 2.695150 & 0.237800 \\
\hline $\mathrm{H}$ & 1.0 & 0.888750 & -0.457350 & 3.289100 \\
\hline $\mathrm{H}$ & 1.0 & 3.691550 & -1.492750 & 0.140800 \\
\hline $\mathrm{H}$ & 1.0 & 4.277650 & -3.592850 & 1.360200 \\
\hline $\mathrm{H}$ & 1.0 & 3.148450 & -4.139850 & 3.527700 \\
\hline $\mathrm{H}$ & 1.0 & 1.455050 & -2.564050 & 4.483800 \\
\hline $\mathrm{H}$ & 1.0 & 2.944050 & 2.785750 & 0.426800 \\
\hline $\mathrm{H}$ & 1.0 & 1.769650 & 4.139850 & 0.493000 \\
\hline $\mathrm{H}$ & 1.0 & 1.813850 & 2.946750 & 1.804800 \\
\hline $\mathrm{H}$ & 1.0 & 0.798750 & 1.475050 & -4.329800 \\
\hline $\mathrm{H}$ & 1.0 & 2.173950 & 2.611650 & -4.119100 \\
\hline $\mathrm{H}$ & 1.0 & 2.478150 & 0.897450 & -4.483800 \\
\hline $\mathrm{H}$ & 1.0 & 0.588850 & -0.523850 & -2.843200 \\
\hline $\mathrm{H}$ & 1.0 & 2.320850 & -0.880650 & -2.761700 \\
\hline $\mathrm{H}$ & 1.0 & 1.422050 & -0.629650 & -1.253100 \\
\hline $\mathrm{H}$ & 1.0 & 3.654250 & 2.487850 & -1.989500 \\
\hline $\mathrm{H}$ & 1.0 & 3.506150 & 1.054150 & -0.91110 \\
\hline $\mathrm{H}$ & 1.0 & 4.020850 & 0.851550 & -2.59230 \\
\hline
\end{tabular}


Product 3 -conformation A

Atom

C 6.0

C 6.0

C 6.0

C 6.0

C 6.0

C 6.0

C 6.0

C 6.0

08.0

$0 \quad 8.0$

08.0

C 6.0

C 6.0

$\mathrm{H} \quad 1.0$

C 6.0

C 6.0

C 6.0

C 6.0

C 6.0

$\mathrm{H} \quad 1.0$

$\mathrm{H} \quad 1.0$

$\mathrm{H} \quad 1.0$

$\mathrm{H} \quad 1.0$

$\mathrm{H} \quad 1.0$

$\mathrm{H} \quad 1.0$

$\mathrm{H} \quad 1.0$

$\mathrm{H} \quad 1.0$

$\mathrm{H} \quad 1.0$

$\mathrm{H} \quad 1.0$

$\mathrm{H} \quad 1.0$

$\mathrm{H} \quad 1.0$

$\mathrm{H} \quad 1.0$

$\mathrm{H} \quad 1.0$

$\mathrm{H} \quad 1.0$

$\mathrm{H} \quad 1.0$

$\mathrm{H} \quad 1.0$

$\mathrm{H} \quad 1.0$

$\mathrm{H} \quad 1.0$

$\mathrm{H} \quad 1.0$

$\mathrm{H} \quad 1.0$

$\mathrm{H} \quad 1.0$

$\mathrm{H} \quad 1.0$
$\mathrm{X}$

$-1.440050$

$-2.657050$

$-3.242350$

$-2.599350$

$-1.382550$

$-0.776450$

0.559650

0.533050

1. 764250

2. 296150

2. 203250

0.679250

$-0.690750$

1. 747450

1. 342350

1. 546750

1. 879050

3. 130150

3. 453450

3.064950

$-1.015250$

$-3.155950$

$-4.201350$

$-3.054450$

$-0.922050$

0.480750

$-0.705950$

$-0.682150$

$-1.656550$

0.642050

2. 400950

1. 460550

0.976850

2.075850

2. 729550

3. 147850

3. 926050

3.469650

3. 902650

4. 201350

2. 315250

3. 956550

2. 625450
$\mathrm{Y}$

Z

$$
0.700450
$$

0.241350

$-0.920850$

$-1.616450$

$-1.150450$

0.018150

0.535050

0.492050

1. 118350

0.308650

2. 206350

1. 911050

1. 215350

$-0.242050$

2. 324250

$-0.292250$

$-1.704550$

0.418950

0.659850

1. 579250

1. 617150

0.796050

$-1.285550$

$-2.535250$

$-1.737450$

$-0.567150$

2. 296950

1.150450

0.774750

$-0.871750$

$-0.791250$

0.726750

$-2.310650$

$-1.771250$

$-2.220950$

1. 491050

$-0.081850$

0.340650

$-0.284650$

1. 128250

1.089050

1. 832750

2. 535250
$-2.980150$

$-3.497150$

$-2.987150$

$-1.959550$

$-1.447750$

$-1.943650$

$-1.391750$

0.167850

0.815850

1. 752550

0.515350

$-1.745350$

0.768650

$-2.078050$

$-1.209050$

$-3.615350$

$-1.593550$

$-1.849750$

2.484450

3. 646650

$-3.419550$

$-4.309950$

$-3.392250$

$-1.552050$

$-0.638950$

0.506150

0.503150

1. 881650

0.433950

$-3.906350$

$-4.127550$

$-4.055850$

$-1.825950$

$-0.500550$

$-2.094350$

$-2.148050$

$-2.446750$

$-0.794950$

2. 876550

1. 801250

4.309950

4.265550

3. 280450 
Product 3 -conformation $B$

Atom

$\mathrm{X}$

$\mathrm{Y}$

Z

\begin{tabular}{|c|c|c|c|c|}
\hline $\mathrm{C}$ & 6.0 & -0.512300 & 1.639850 & -2.091250 \\
\hline $\mathrm{C}$ & 6.0 & -1.798200 & 1.919350 & -2.568650 \\
\hline $\mathrm{C}$ & 6.0 & -2.882400 & 1.131150 & -2.173850 \\
\hline C & 6.0 & -2.664200 & 0.061950 & -1.301750 \\
\hline C & 6.0 & -1.374600 & -0.206250 & -0.828250 \\
\hline C & 6.0 & -0.268100 & 0.573350 & -1.204950 \\
\hline C & 6.0 & 1.140600 & 0.282350 & -0.668550 \\
\hline C & 6.0 & 1.125500 & 0.225950 & 0.899050 \\
\hline C & 6.0 & 0.294400 & 1.413850 & 1.394050 \\
\hline 0 & 8.0 & -0.757900 & 1.038450 & 2.145450 \\
\hline O & 8.0 & 0.546400 & 2.562450 & 1.100850 \\
\hline 0 & 8.0 & 1.982000 & 1.407850 & -0.944950 \\
\hline C & 6.0 & 0.857500 & -1.083450 & 1.664650 \\
\hline $\mathrm{C}$ & 6.0 & 1.805600 & -0.908050 & -1.451250 \\
\hline $\mathrm{H}$ & 1.0 & 1.605800 & 2.177150 & -0.536850 \\
\hline $\mathrm{C}$ & 6.0 & 1.957600 & -0.546250 & -2.955250 \\
\hline C & 6.0 & 0.994400 & -2.224050 & -1.439650 \\
\hline C & 6.0 & 3.233200 & -1.199750 & -0.929850 \\
\hline $\mathrm{C}$ & 6.0 & -1.687500 & 1.969850 & 2.663250 \\
\hline $\mathrm{C}$ & 6.0 & -2.716600 & 2.319150 & 1.583250 \\
\hline $\mathrm{H}$ & 1.0 & 0.298400 & 2.295750 & -2.444750 \\
\hline $\mathrm{H}$ & 1.0 & -1.959200 & 2.764250 & -3.259750 \\
\hline $\mathrm{H}$ & 1.0 & -3.897100 & 1.349150 & -2.547450 \\
\hline $\mathrm{H}$ & 1.0 & -3.512000 & -0.569450 & -0.985550 \\
\hline $\mathrm{H}$ & 1.0 & -1.263400 & -1.060550 & -0.149350 \\
\hline $\mathrm{H}$ & 1.0 & 2.158100 & 0.481250 & 1.241650 \\
\hline $\mathrm{H}$ & 1.0 & -0.145800 & -1.522450 & 1.484950 \\
\hline $\mathrm{H}$ & 1.0 & 0.925900 & -0.912450 & 2.764450 \\
\hline $\mathrm{H}$ & 1.0 & 1.613600 & -1.871550 & 1.460650 \\
\hline $\mathrm{H}$ & 1.0 & 0.976600 & -0.321350 & -3.431050 \\
\hline $\mathrm{H}$ & 1.0 & 2.407000 & -1.384650 & -3.535150 \\
\hline $\mathrm{H}$ & 1.0 & 2.622000 & 0.330950 & -3.123750 \\
\hline $\mathrm{H}$ & 1.0 & 0.022400 & -2.123250 & -1.971650 \\
\hline $\mathrm{H}$ & 1.0 & 0.784900 & -2.606650 & -0.420650 \\
\hline $\mathrm{H}$ & 1.0 & 1.542900 & -3.040850 & -1.961850 \\
\hline $\mathrm{H}$ & 1.0 & 3.897100 & -0.309350 & -1.005950 \\
\hline $\mathrm{H}$ & 1.0 & 3.723300 & -2.011250 & -1.514750 \\
\hline $\mathrm{H}$ & 1.0 & 3.245300 & -1.527550 & 0.130750 \\
\hline $\mathrm{H}$ & 1.0 & -2.195400 & 1.490650 & 3.53515 \\
\hline $\mathrm{H}$ & 1.0 & -1.146800 & 2.868750 & 3.043650 \\
\hline $\mathrm{H}$ & 1.0 & -3.261100 & 1.408450 & 1.243250 \\
\hline $\mathrm{H}$ & 1.0 & -3.470700 & 3.040850 & 1.973350 \\
\hline $\mathrm{H}$ & 1.0 & -2.233400 & 2.783250 & 0.692750 \\
\hline
\end{tabular}


Product 3 -conformation C

Atom

$\mathrm{X}$

$\mathrm{Y}$

Z

\begin{tabular}{|c|c|c|c|c|}
\hline C & 6.0 & -1.137850 & 2.471000 & -1.879150 \\
\hline $\mathrm{C}$ & 6.0 & -2.440950 & 2.282000 & -2.354750 \\
\hline C & 6.0 & -3.129050 & 1.098800 & -2.073050 \\
\hline C & 6.0 & -2.497050 & 0.109500 & -1.316250 \\
\hline C & 6.0 & -1.193350 & 0.309500 & -0.847250 \\
\hline C & 6.0 & -0.480250 & 1.491900 & -1.108550 \\
\hline C & 6.0 & 0.949150 & 1.707300 & -0.578450 \\
\hline C & 6.0 & 0.950050 & 1.600100 & 0.988150 \\
\hline $\mathrm{C}$ & 6.0 & 0.865850 & 0.234100 & 1.666950 \\
\hline O & 8.0 & 2.089150 & -0.281400 & 1.895450 \\
\hline O & 8.0 & -0.162750 & -0.302200 & 2.016050 \\
\hline O & 8.0 & 1.321950 & 3.061700 & -0.831550 \\
\hline C & 6.0 & 1.971350 & 2.478900 & 1.750350 \\
\hline C & 6.0 & 1.972750 & 0.852600 & -1.417350 \\
\hline $\mathrm{H}$ & 1.0 & 0.691750 & 3.625600 & -0.414150 \\
\hline $\mathrm{C}$ & 6.0 & 1.931850 & 1.282800 & -2.910750 \\
\hline $\mathrm{C}$ & 6.0 & 1.699850 & -0.667600 & -1.427050 \\
\hline C & 6.0 & 3.433450 & 1.058800 & -0.953650 \\
\hline C & 6.0 & 2.293550 & -1.454200 & 2.659950 \\
\hline C & 6.0 & 2.119450 & -2.700000 & 1.786350 \\
\hline $\mathrm{H}$ & 1.0 & -0.655550 & 3.424400 & -2.144650 \\
\hline $\mathrm{H}$ & 1.0 & -2.929650 & 3.068000 & -2.955450 \\
\hline $\mathrm{H}$ & 1.0 & -4.156250 & 0.946800 & -2.445350 \\
\hline $\mathrm{H}$ & 1.0 & -3.027550 & -0.831300 & -1.090350 \\
\hline $\mathrm{H}$ & 1.0 & -0.747050 & -0.509100 & -0.268050 \\
\hline $\mathrm{H}$ & 1.0 & -0.019050 & 2.071100 & 1.284950 \\
\hline $\mathrm{H}$ & 1.0 & 3.023950 & 2.258000 & 1.471150 \\
\hline $\mathrm{H}$ & 1.0 & 1.889850 & 2.325900 & 2.851950 \\
\hline $\mathrm{H}$ & 1.0 & 1.806950 & 3.569700 & 1.592050 \\
\hline $\mathrm{H}$ & 1.0 & 0.914150 & 1.176700 & -3.349250 \\
\hline $\mathrm{H}$ & 1.0 & 2.610850 & 0.659200 & -3.536450 \\
\hline $\mathrm{H}$ & 1.0 & 2.256150 & 2.337000 & -3.062650 \\
\hline $\mathrm{H}$ & 1.0 & 0.786150 & -0.934000 & -2.002950 \\
\hline $\mathrm{H}$ & 1.0 & 1.599450 & -1.081900 & -0.404650 \\
\hline $\mathrm{H}$ & 1.0 & 2.530350 & -1.230100 & -1.911350 \\
\hline $\mathrm{H}$ & 1.0 & 3.719950 & 2.133900 & -0.921050 \\
\hline $\mathrm{H}$ & 1.0 & 4.156250 & 0.563600 & -1.641550 \\
\hline $\mathrm{H}$ & 1.0 & 3.621650 & 0.615400 & 0.046250 \\
\hline $\mathrm{H}$ & 1.0 & 3.335750 & -1.414100 & 3.059950 \\
\hline $\mathrm{H}$ & 1.0 & 1.602750 & -1.452400 & 3.536450 \\
\hline $\mathrm{H}$ & 1.0 & 2.841850 & -2.698500 & 0.937750 \\
\hline $\mathrm{H}$ & 1.0 & 2.299350 & -3.625600 & 2.380550 \\
\hline $\mathrm{H}$ & 1.0 & 1.088550 & -2.765500 & 1.368850 \\
\hline
\end{tabular}



-conformation A

$\begin{array}{rrrrr}\mathrm{C} & 6.0 & -1.940050 & 0.891650 & -2.473150 \\ \mathrm{C} & 6.0 & -2.834650 & 0.555450 & -3.495750 \\ \mathrm{C} & 6.0 & -2.830450 & -0.733950 & -4.035750 \\ \mathrm{C} & 6.0 & -1.923850 & -1.678750 & -3.546550 \\ \mathrm{C} & 6.0 & -1.031950 & -1.334850 & -2.523550 \\ \mathrm{C} & 6.0 & -1.024750 & -0.046050 & -1.961950 \\ \mathrm{C} & 6.0 & -0.051850 & 0.339950 & -0.847150 \\ \mathrm{C} & 6.0 & 0.036650 & -0.764450 & 0.247450 \\ \mathrm{C} & 6.0 & 1.033050 & -0.396250 & 1.344350 \\ \mathrm{O} & 8.0 & 1.807650 & -1.446950 & 1.678550 \\ \mathrm{O} & 8.0 & 1.114650 & 0.705950 & 1.839350 \\ \mathrm{O} & 8.0 & -0.537350 & 1.507650 & -0.187950 \\ \mathrm{C} & 6.0 & -1.321050 & -1.069750 & 0.907050 \\ \mathrm{C} & 6.0 & 1.338650 & 0.708750 & -1.451150 \\ \mathrm{H} & 1.0 & -0.010250 & 1.662650 & 0.585650 \\ \mathrm{C} & 6.0 & 1.245150 & 1.780050 & -2.557150 \\ \mathrm{C} & 6.0 & 2.155450 & -0.479650 & -1.991850 \\ \mathrm{C} & 6.0 & 2.821050 & -1.356050 & 2.660050 \\ \mathrm{C} & 6.0 & 2.199850 & -1.501650 & 4.052850 \\ \mathrm{H} & 1.0 & -1.974550 & 1.921250 & -2.082550 \\ \mathrm{H} & 1.0 & -3.544250 & 1.308750 & -3.878450 \\ \mathrm{H} & 1.0 & -3.534350 & -1.002350 & -4.841550 \\ \mathrm{H} & 1.0 & -1.912450 & -2.697450 & -3.970250 \\ \mathrm{H} & 1.0 & -0.336450 & -2.114950 & -2.179350 \\ \mathrm{H} & 1.0 & 0.393550 & -1.712650 & -0.213850 \\ \mathrm{H} & 1.0 & -1.719850 & -0.193250 & 1.466650 \\ \mathrm{H} & 1.0 & -1.227250 & -1.907750 & 1.636150 \\ \mathrm{H} & 1.0 & -2.087750 & -1.372350 & 0.158150 \\ \mathrm{H} & 1.0 & 1.950850 & 1.156450 & -0.630550 \\ \mathrm{H} & 1.0 & 0.715150 & 1.395050 & -3.457650 \\ \mathrm{H} & 1.0 & 0.715750 & 2.697450 & -2.213150 \\ \mathrm{H} & 1.0 & 2.258350 & 2.104350 & -2.889150 \\ \mathrm{H} & 1.0 & 1.655850 & -0.964550 & -2.859450 \\ \mathrm{H} & 1.0 & 3.157650 & -0.144550 & -2.345750 \\ \mathrm{H} & 1.0 & 2.348050 & -1.254550 & -1.216850 \\ \mathrm{H} & 1.0 & 3.375650 & -0.395350 & 2.538150 \\ \mathrm{H} & 1.0 & 3.544250 & -2.186950 & 2.474550 \\ \mathrm{H} & 1.0 & 1.482850 & -0.676250 & 4.267550 \\ \mathrm{H} & 1.0 & 2.987050 & -1.478750 & 4.841550 \\ \mathrm{H} & 1.0 & 1.649650 & -2.466550 & 4.145650\end{array}$


Product 4 -conformation B Atom $\mathrm{X}$ Y Z

$\begin{array}{lrrrr}\mathrm{C} & 6.0 & -0.792150 & 0.537550 & -2.365000 \\ \mathrm{C} & 6.0 & -1.652450 & -0.391350 & -2.961600 \\ \mathrm{C} & 6.0 & -1.827750 & -1.654850 & -2.390200 \\ \mathrm{C} & 6.0 & -1.130850 & -1.978250 & -1.223500 \\ \mathrm{C} & 6.0 & -0.272650 & -1.041750 & -0.634900 \\ \mathrm{C} & 6.0 & -0.088650 & 0.238350 & -1.183500 \\ \mathrm{C} & 6.0 & 0.845250 & 1.267050 & -0.540100 \\ \mathrm{C} & 6.0 & 0.830550 & 1.230850 & 1.025800 \\ \mathrm{C} & 6.0 & -0.619550 & 1.133350 & 1.498500 \\ \mathrm{O} & 8.0 & -0.810650 & 0.146950 & 2.394200 \\ \mathrm{O} & 8.0 & -1.497150 & 1.855650 & 1.077900 \\ \mathrm{O} & 8.0 & 0.365750 & 2.577450 & -0.850600 \\ \mathrm{C} & 6.0 & 1.842150 & 0.344250 & 1.777000 \\ \mathrm{C} & 6.0 & 2.273650 & 1.160750 & -1.156200 \\ \mathrm{H} & 1.0 & -0.509850 & 2.655950 & -0.491800 \\ \mathrm{C} & 6.0 & 2.416650 & 1.861650 & -2.525200 \\ \mathrm{C} & 6.0 & 2.782950 & -0.283850 & -1.332400 \\ \mathrm{C} & 6.0 & -2.085650 & -0.154450 & 2.926200 \\ \mathrm{C} & 6.0 & -2.856350 & -1.034850 & 1.936900 \\ \mathrm{H} & 1.0 & -0.688550 & 1.515550 & -2.860800 \\ \mathrm{H} & 1.0 & -2.194450 & -0.127950 & -3.885800 \\ \mathrm{H} & 1.0 & -2.506150 & -2.389050 & -2.856800 \\ \mathrm{H} & 1.0 & -1.258750 & -2.974550 & -0.766900 \\ \mathrm{H} & 1.0 & 0.252650 & -1.353450 & 0.276300 \\ \mathrm{H} & 1.0 & 1.131950 & 2.249950 & 1.372300 \\ \mathrm{H} & 1.0 & 1.688850 & -0.745250 & 1.626800 \\ \mathrm{H} & 1.0 & 1.782050 & 0.517950 & 2.876900 \\ \mathrm{H} & 1.0 & 2.895250 & 0.578850 & 1.503300 \\ \mathrm{H} & 1.0 & 2.971250 & 1.703850 & -0.473800 \\ \mathrm{H} & 1.0 & 1.740550 & 1.413950 & -3.287600 \\ \mathrm{H} & 1.0 & 2.203950 & 2.953650 & -2.470600 \\ \mathrm{H} & 1.0 & 3.456250 & 1.772350 & -2.916800 \\ \mathrm{H} & 1.0 & 2.211050 & -0.827250 & -2.118600 \\ \mathrm{H} & 1.0 & 3.853150 & -0.295150 & -1.642400 \\ \mathrm{H} & 1.0 & 2.721750 & -0.885750 & -0.401200 \\ \mathrm{H} & 1.0 & -2.626250 & 0.791650 & 3.166300 \\ \mathrm{H} & 1.0 & -1.929150 & -0.704350 & 3.885800 \\ \mathrm{H} & 1.0 & -3.026850 & -0.509050 & 0.969300 \\ \mathrm{H} & 1.0 & -3.853150 & -1.314750 & 2.349200 \\ \mathrm{H} & 1.0 & -2.298950 & -1.975850 & 1.722600 \\ & & & & \end{array}$


-conformation C

Atom

$\mathrm{X}$

$\mathrm{Y}$

Z

$\begin{array}{rrrrr}\mathrm{C} & 6.0 & -1.411450 & 1.132300 & -2.086200 \\ \mathrm{C} & 6.0 & -2.594850 & 0.571700 & -2.581300 \\ \mathrm{C} & 6.0 & -2.785550 & -0.812500 & -2.547700 \\ \mathrm{C} & 6.0 & -1.784550 & -1.626600 & -2.011500 \\ \mathrm{C} & 6.0 & -0.604550 & -1.056400 & -1.519800 \\ \mathrm{C} & 6.0 & -0.385950 & 0.331500 & -1.550800 \\ \mathrm{C} & 6.0 & 0.904350 & 0.952200 & -1.009100 \\ \mathrm{C} & 6.0 & 0.915550 & 0.891600 & 0.550400 \\ \mathrm{C} & 6.0 & 0.460450 & -0.423600 & 1.177000 \\ \mathrm{O} & 8.0 & -0.884750 & -0.462800 & 1.283900 \\ \mathrm{O} & 8.0 & 1.193650 & -1.308500 & 1.557500 \\ \mathrm{O} & 8.0 & 0.888350 & 2.352500 & -1.305000 \\ \mathrm{C} & 6.0 & 2.235850 & 1.364500 & 1.188000 \\ \mathrm{C} & 6.0 & 2.176650 & 0.424700 & -1.748800 \\ \mathrm{H} & 1.0 & 1.730650 & 2.719800 & -1.098900 \\ \mathrm{C} & 6.0 & 2.049850 & 0.557100 & -3.282500 \\ \mathrm{C} & 6.0 & 2.682650 & -0.996200 & -1.443300 \\ \mathrm{C} & 6.0 & -1.574150 & -1.574100 & 1.820800 \\ \mathrm{C} & 6.0 & -1.579250 & -1.485400 & 3.349800 \\ \mathrm{H} & 1.0 & -1.318050 & 2.228800 & -2.133100 \\ \mathrm{H} & 1.0 & -3.381650 & 1.222900 & -2.998800 \\ \mathrm{H} & 1.0 & -3.717150 & -1.256900 & -2.936700 \\ \mathrm{H} & 1.0 & -1.928050 & -2.719800 & -1.972600 \\ \mathrm{H} & 1.0 & 0.140650 & -1.741000 & -1.092300 \\ \mathrm{H} & 1.0 & 0.153950 & 1.628000 & 0.908100 \\ \mathrm{H} & 1.0 & 3.088550 & 0.691900 & 0.946800 \\ \mathrm{H} & 1.0 & 2.152850 & 1.396400 & 2.299400 \\ \mathrm{H} & 1.0 & 2.512750 & 2.391200 & 0.856400 \\ \mathrm{H} & 1.0 & 3.017150 & 1.095900 & -1.445000 \\ \mathrm{H} & 1.0 & 1.242550 & -0.097700 & -3.682200 \\ \mathrm{H} & 1.0 & 1.830150 & 1.599500 & -3.606400 \\ \mathrm{H} & 1.0 & 2.995950 & 0.262900 & -3.792800 \\ \mathrm{H} & 1.0 & 2.074150 & -1.786300 & -1.933800 \\ \mathrm{H} & 1.0 & 3.717150 & -1.136000 & -1.834700 \\ \mathrm{H} & 1.0 & 2.738250 & -1.210900 & -0.356700 \\ \mathrm{H} & 1.0 & -1.110750 & -2.518700 & 1.448700 \\ \mathrm{H} & 1.0 & -2.620250 & -1.540000 & 1.430300 \\ \mathrm{H} & 1.0 & -0.545850 & -1.530100 & 3.763700 \\ \mathrm{H} & 1.0 & -2.157350 & -2.329000 & 3.792800 \\ \mathrm{H} & 1.0 & -2.045550 & -0.532900 & 3.692700\end{array}$

